\title{
Congenital Esophageal Duplication Cyst: A Rare Cause of Dysphagia in an Adult
}

\author{
Nikhil Sonthaliaa, b, Samit S. Jain ${ }^{\mathrm{a}}$, Ravindra G. Surude ${ }^{\mathrm{a}}$, Ashok R. Mohite ${ }^{\mathrm{a}}$, \\ Pravin M. Rathi ${ }^{\mathrm{a}}$
}

\begin{abstract}
Esophageal duplication cyst is a rare congenital embryonal gastrointestinal (GI) malformation which is diagnosed most commonly in childhood. In adults, they can present with a variety of symptoms ranging from dysphagia, chest pain, epigastric discomfort, and vomiting to more serious complications including infections, hemorrhage, and ulcerations. A 30-year-old male presented with gradually progressive dysphagia to solids for 4 months without significant weight loss. Clinical examination and routine laboratory examination were unrevealing. Upper GI endoscopy revealed a well-defined submucosal lesion bulging into the esophageal lumen involving the right antero-lateral wall of the distal esophagus. The overlying mucosa was normal with mild luminal narrowing but gastroscope could be negotiated across this narrowing. Differential diagnosis included lipoma, leiomyoma or GI stromal tumors. Contrast-enhanced computed tomography of thorax revealed a $3.5 \times 2.3 \times 3 \mathrm{~cm}$ well-defined homogenous hypodense lesion involving the right antero-lateral wall of the distal thoracic esophagus with likely possibility of submucosal or intramural lesion. Subsequently, endoscopic ultrasonography (EUS) revealed a $3.3 \times 2.8 \mathrm{~cm}$ homogenous hypoechoic lesion without any vascularity involving the distal esophagus wall suggestive of duplication cyst. The lesion was intramural in location as muscularis propria was seen to go around the lesion. Bronchogenic cyst was excluded due to absence of cartilage and close proximity of the cyst to lumen. Fine-needle aspiration was not attempted in view of high risk of introducing infection. Being symptomatic, the patient underwent complete surgical excision of the cyst with exteriorization of the base which on histopathology confirmed duplication cyst. Esophageal duplication cysts are exceedingly rare congenital embryonal malformations with estimated prevalence of $0.0122 \%$ arising from aberration of posterior division of embryonic foregut at 3 - 4 weeks of gestation. This case shows that duplication cysts can rarely masquerade as submucosal tumor in adults and EUS is highly accurate in pre-operative diagnosis
\end{abstract}

Manuscript accepted for publication August 16, 2016

aDepartment of Gastroenterology, Topiwala National Medical College and BYL Nair Ch Hospital, Dr. A.L Nair Road, Mumbai 400008, Maharashtra, India ${ }^{b}$ Corresponding Author: Nikhil Sonthalia, Department of Gastroenterology, Topiwala National Medical College and BYL Nair Ch Hospital, Dr. A.L Nair Road, Mumbai 400008, Maharashtra, India. Email: nikhil_zenith@yahoo.co.in

doi: http://dx.doi.org/10.14740/gr711w and therapeutic decision making. Literature search revealed only a handful of cases of EUS-guided diagnosis of esophageal duplication cyst reported in the literature.

Keywords: Duplication cyst; Submucosal tumor; Dysphagia

\section{Introduction}

Gastrointestinal (GI) duplication cysts are rare entities which occur most commonly in ileum, jejunum, esophagus, and colon. Esophageal duplication cyst accounts for $10-15 \%$ of all congenital duplication cysts involving the GI tract [1]. Majority of these cysts are diagnosed in childhood but when present in adults they are more likely to be symptomatic [2]. Most common location is the right postero-inferior mediastinum with about two-third being located in the lower esophagus [3]. When symptomatic, they can manifest a wide range of symptoms ranging from dysphagia, chest pain, epigastric discomfort, and vomiting to stridor and non-productive cough depending upon the location of the cyst. We herein report a case of young adult presenting with dysphagia who posed a diagnostic dilemma on upper GI endoscopy as the lesion mimicked as submucosal tumors like lipoma, leiomyoma and GI stromal tumors. Endoscopic ultrasound (EUS) and subsequent histopathology following surgical resection confirmed the diagnosis of esophageal duplication cyst in our case, highlighting the indispensable role of EUS in managing these patients. There is a paucity of data in the literature regarding this rare congenital malformation presenting symptomatically in an adult.

\section{Case Report}

A 30-year-old male presented to us with the complaints of gradually progressive dysphagia mainly to solids for 4 months' duration. He denied any history of significant weight loss, vomiting, acute abdominal pain, abdominal distension, hematemesis, melena, dyspnea on exertion, facial puffiness or peripheral edema. The patient denied any history of addiction. On physical examination, his vitals were stable with pulse rate of $90 / \mathrm{min}$ and blood pressure of 120/70 mm Hg. Rest of the abdominal and respiratory examinations were within normal limits. 


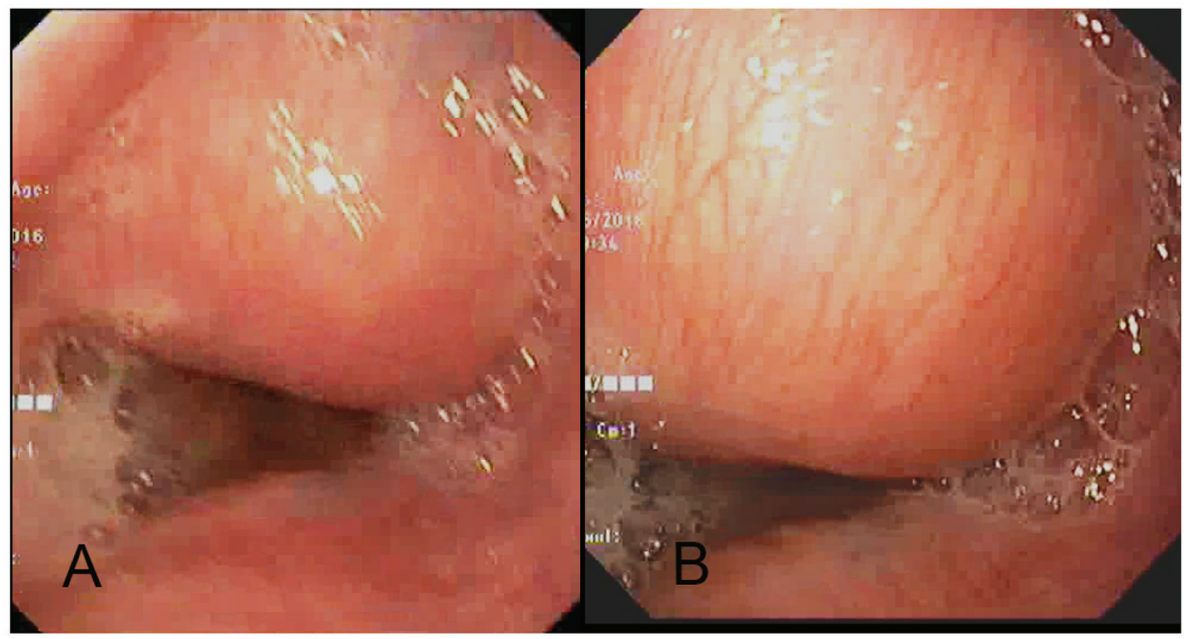

Figure 1. (A, B) Upper gastrointestinal endoscopy image shows a submucosal lesion in the right antero-lateral wall of the distal thoracic esophagus which is bulging into the esophageal lumen with normal overlying mucosa and mild luminal narrowing.

On further evaluation, his hematological and biochemical parameters were within normal limits. Upper GI endoscopy revealed a well-defined submucosal lesion which was bulging into the esophageal lumen involving the right antero-lateral wall of the distal esophagus just above the gastro-esophageal junction (Fig. 1A, B). The overlying mucosa was normal and there was mild luminal narrowing but gastroscope could be negotiated across this narrowing. Ultrasonography of the abdomen was unrevealing. Further contrast-enhanced computed tomography $(\mathrm{CT})$ imaging of the thorax revealed a $3.5 \times 2.3$ $\times 3 \mathrm{~cm}$ well-defined homogenous hypodense cystic lesion involving the right antero-lateral wall of the distal thoracic esophagus with likely possibility of submucosal or intramural lesion (Fig. 2). The patient was then subjected to EUS which revealed a $3.3 \times 2.8 \mathrm{~cm}$ homogenous hypoechoic lesion without any vascularity involving the distal esophagus wall (Fig.

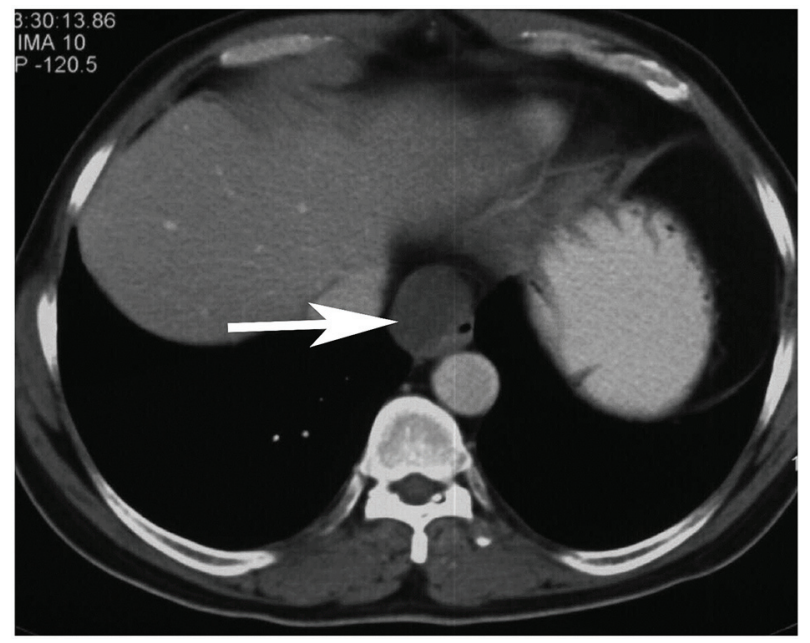

Figure 2. Contrast-enhanced computed tomography image of the thorax shows a $3.5 \times 2.3 \times 3 \mathrm{~cm}$ well-defined homogenous cystic lesion (white arrow) along the right antero-lateral aspect of the distal esophagus focally indenting and distorting the lumen.
$3 \mathrm{~A}, \mathrm{~B})$. The lesion was intramural in location as muscularis propria was seen to go around the lesion. It could be easily identified as a duplication cyst rather than a bronchogenic cyst as there was absence of cartilage and the lesion was in close proximity to the esophageal lumen. In view of imaging finding consistent with that of duplication cyst, fine-needle aspiration (FNA)-guided fluid aspiration was not attempted in this patient in view of high risk of introducing infection. In view of the cyst being symptomatic, the patient underwent complete surgical excision of the cyst with exteriorization of the base. On histopathology, the lesion showed features consistent with esophageal duplication cyst. At present, the patient is asymptomatic on 6-month follow-up.

\section{Discussion}

Esophageal duplication cysts are exceedingly rare congenital embryonal malformations with estimated prevalence of $0.0122 \%$ [1]. They arise from aberration of posterior division of embryonic foregut at 3 - 4 weeks of gestation [4]. Majority of them are found in the mediastinum where they present either as separate masses along or in continuity with native esophagus. Intramural esophageal cysts as seen in our case are considered very uncommon in adults $[5,6]$. They have a double layer of surrounding esophageal smooth muscle and are lined by either squamous or enteric epithelium. They are most commonly found in lower esophagus; however, cases of intraabdominal esophageal duplication cysts have also been reported [7]. Duplication cysts may be cystic, tubular or present as a diverticulum with cystic type being more common. In our case, there was a cystic type duplication cyst present in right antero-lateral wall of the distal esophagus.

Though commonly diagnosed in childhood, esophageal duplication cysts are rare but more symptomatic in adults [2]. They may present with dysphagia, chest pain, epigastric discomfort, vomiting, stridor, cough, bleeding, and hematemesis or may be incidentally detected. In our case, the patient pre- 


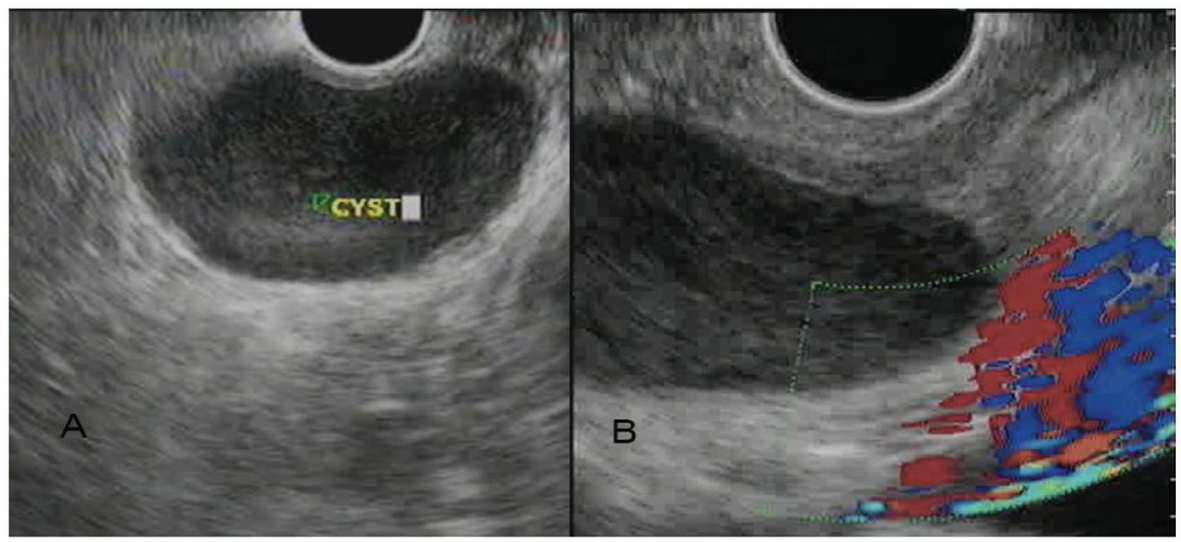

Figure 3. Endoscopic ultrasound image of the patient shows a $3.3 \times 2.8 \mathrm{~cm}$ homogenous hypoechoic intramural lesion with well-defined margins involving the distal esophageal wall (A). The lesion did not show any vascularity on Doppler imaging (B).

sented with dysphagia due to compression into the esophageal lumen. Most of the case reports in the literature of symptomatic duplication cysts are of children [8].

On routine esophagogastroduodenoscopy, these cysts may present as an extrinsic compression into the esophageal lumen or as submucosal lesions with normal overlying mucosa. Differential diagnosis to be considered for these lesions on upper GI endoscopy includes lipomas, leiomyomas, and GI stromal tumors. Rarely, a separate opening communicating with the esophageal lumen may be seen in cases of tubular or diverticulum type duplication cysts. Endoscopy-guided mucosal biopsy is not yielding in these cases. Other differential diagnosis that needs to be considered in these cases includes other mediastinal cysts like hydatid cyst, pericardial cysts, bronchogenic cysts, or mediastinal lymphadenopathy. CT images of thorax in cases of esophageal duplication cysts usually reveal a homogenous low attenuating cystic lesion with well-defined margins along the wall of esophagus causing variable lumen compromise.

EUS has proved to be imaging modality of choice for characterization of mediastinal cysts and lymph nodes. EUS is indispensable in distinguishing esophageal duplication cyst from bronchogenic cyst by their proximity to the esophagus, absence of cartilage and presence of double muscle layer around them $[8,9]$. On EUS, it usually presents as a periesophageal homogenous hypoechoic mass with multi-layered wall and well-defined margin [10]. The muscle layer of the cyst is in continuation with the muscularis propria of the esophagus. The cyst may be completely anechoic if there is significant central fluid. It may also be heteroechoic with anechoic areas if significant pus or blood is present. The role of EUS-guided FNA of the cystic fluid content remains controversial in view of high risk of introducing infection. One of the studies showed infection rates up to $14 \%$ following FNA despite adequate use of pre- and post-procedure antibiotics [11]. EUS-guided FNA should be considered in cases with indeterminate diagnosis, where imaging is atypical for duplication cysts or where there is concern for malignancy. EUS-guided FNA of the esophageal duplication cysts usually reveals squamous cells with cystic fluid content without any atypical cells.

Complete cyst excision/cyst enucleation is the treatment of choice in symptomatic cases which is curative. In asymptomatic patient, role of surgery is not clearly defined as surgical intervention can lead to long-term complications like heartburn and reflux esophagitis and carry a mortality rate of approximately $1 \%$ [12]. Few case reports have advocated surveillance programs using periodic EUS for asymptomatic cysts but exact duration and frequency of EUS as well as costeffectiveness of this approach is not well defined in the literature [13].

\section{Author Contributions}

All authors contributed equally to the manuscript. Nikhil Sonthalia and Ashok R. Mohite designed and wrote the manuscript. Samit Jain and Nikhil Sonthalia evaluated the patient, did the literature search and recorded clinical findings. Ravindra Surude and Pravin M. Rathi conceptualized the manuscript, gave the intellectual input and did critical review of the manuscript.

\section{Conflicts of Interest}

Authors have no conflicts of interest to be declared.

\section{Consent}

Informed consent was taken from the patient.

\section{Disclosure}

This case report is exempted from the institutional ethics committee approval.

\section{Grants/Funding}

Authors have received no grants or funding for this case report. 


\section{References}

1. Olajide AR, Yisau AA, Abdulraseed NA, Kashim IO, Olaniyi AJ, Morohunfade AO. Gastrointestinal duplications: Experience in seven children and a review of the literature. Saudi J Gastroenterol. 2010;16(2):105-109.

2. Karnak I, Ocal T, Senocak ME, Tanyel FC, Buyukpamuk$\mathrm{cu}$ N. Alimentary tract duplications in children: report of 26 years' experience. Turk J Pediatr. 2000;42(2):118-125.

3. Bhatia V, Tajika M, Rastogi A. Upper gastrointestinal submucosal lesions - clinical and endosonographic evaluation and management. Trop Gastroenterol. 2010;31(1):529.

4. Eichmann D, Engler S, Oldigs HD, Schroeder H, Partsch CJ. Radiological case of the month. Denouement and discussion: congenital esophageal duplication cyst as a rare cause of neonatal progressive stridor. Arch Pediatr Adolesc Med. 2001;155(9):1067-1068.

5. Oteiza F. Congenital esophageal cysts - two cases in adult patients. Hepatogastroenterology. 1999;46:2405-2408.

6. Cioffi U, Bonavina L, De Simone M, Santambrogio L, Pavoni G, Testori A, Peracchia A. Presentation and surgical management of bronchogenic and esophageal duplication cysts in adults. Chest. 1998;113(6):1492-1496.

7. Martin ND, Kim JC, Verma SK, Rubin R, Mitchell DG,
Bergin D, Yeo CJ. Intra-abdominal esophageal duplication cysts: a review. J Gastrointest Surg. 2007;11(6):773777.

8. Wiechowska-Kozlowska A, Wunsch E, Majewski M, Milkiewicz P. Esophageal duplication cysts: endosonographic findings in asymptomatic patients. World J Gastroenterol. 2012;18(11):1270-1272.

9. Kuhlman JE, Fishman EK, Wang KP, Siegelman SS. Esophageal duplication cyst: CT and transesophageal needle aspiration. AJR Am J Roentgenol. 1985;145(3):531532.

10. Diehl DL, Cheruvattath R, Facktor MA, Go BD. Infection after endoscopic ultrasound-guided aspiration of mediastinal cysts. Interact Cardiovasc Thorac Surg. 2010;10(2):338-340.

11. Wildi SM, Hoda RS, Fickling W, Schmulewitz N, Varadarajulu S, Roberts SS, Ferguson B, et al. Diagnosis of benign cysts of the mediastinum: the role and risks of EUS and FNA. Gastrointest Endosc. 2003;58(3):362368.

12. Salo JA, Ala-Kulju KV. Congenital esophageal cysts in adults. Ann Thorac Surg. 1987;44(2):135-138.

13. Versleijen MW, Drenth JP, Nagengast FM. A case of esophageal duplication cyst with a 13-year follow-up period. Endoscopy. 2005;37(9):870-872. 